\title{
Cytomegalovirus seropositivity and incident ischaemic heart disease in the Caerphilly prospective heart disease study
}

\author{
D P Strachan, D Carrington, M A Mendall, B K Butland, P M Sweetnam, P C Elwood
}

Department of Public Health Sciences, St George's Hospital Medical School, Cranmer Terrace, London SW17 0RE, UK D P Strachan B K Butland

Department of Medical Microbiology, St George's Hospital Medical School

D Carrington

Division of Gastroenterology, Endocrinology and Metabolism, St George's Hospital Medical School M A Mendall

Medical Research Council Epidemiology Unit (South Wales), Llandough Hospital, Penarth, South Glamorgan CF64 2XW, UK

P M Sweetnam

P C Elwood

Correspondence to: Professor Strachan email: d.strachan@ sghms.ac.uk

Accepted for publication 19 November 1998

\begin{abstract}
Objective-To assess the role of cytomegalovirus (CMV) infection in primary ischaemic heart disease.

Methods-Plasma specimens collected during 1979-83 from men in Caerphilly, south Wales, were analysed for IgG antibodies to CMV by enzyme linked immunosorbent assay and latex tests. Incident ischaemic heart disease events were ascertained after five and 10 years from death certificates, hospital records, and ECG changes; 195 incident ischaemic heart disease cases were compared with 216 controls of a similar age drawn from the rest of the cohort.
\end{abstract}

Results-164 cases (84\%) and 180 controls (83\%) were seropositive for CMV. Optical density, an indicator of CMV antibody titre, was similar for cases and controls. Among controls, seropositivity was not associated with age, socioeconomic status currently or in childhood, smoking, height, body mass index, blood pressure, total cholesterol, fibrinogen, plasma viscosity, or leucocyte count. The unadjusted odds ratio relating CMV seropositivity to incident ischaemic heart disease was $\mathbf{1 . 0 6}$ (95\% confidence interval 0.63 to 1.79$)$ and was little changed $(1.11,0.63$ to 1.97$)$ after adjustment for age, smoking, body mass index, systolic blood pressure, total cholesterol, and socioeconomic status currently and in childhood.

Conclusions-CMV infection is unlikely to be a strong risk factor for development of myocardial infarction in middle aged men.

(Heart 1999;81:248-251)

Keywords: cytomegalovirus; coronary artery disease; atherosclerosis

Following the identification of cytomegalovirus (CMV) antigen by immunocytochemistry in arterial smooth muscle cells in $1983,{ }^{1} \mathrm{CMV}$ DNA has been detected by in situ hybridisation or polymerase chain reaction in the vascular tissue of patients requiring surgery for symptomatic atherosclerosis, ${ }^{23}$ although it is not localised to the atheromatous plaques ${ }^{3}$ and there is no clear correlation between the distribution of CMV genomic material and that of atheroma. ${ }^{4}$ The presence of CMV genomic sequences in arterial walls is usually associated with CMV seropositivity. ${ }^{3}$ High CMV IgG titres are more common in patients requiring surgery for arteriosclerosis (mainly carotid bypass). ${ }^{5}$ Population based studies of ultrasonographically defined carotid atheroma also suggest an association with CMV seropositivity. $^{67}$

These observations linking CMV infection to arterial disease have led to speculation that CMV may be implicated in coronary atheroma. As a recent review has pointed out, ${ }^{8}$ most of the evidence relates to the development of accelerated coronary artery disease during immunosuppression in transplanted human hearts, or to coronary restenosis after balloon angioplasty. However, there are important histological differences between these secondary lesions and primary coronary atheroma. Whereas CMV DNA was detected in 11 of 24 restenoses after angioplasty, none was detected in 11 primary lesions. ${ }^{9}$ The CMV DNA found in transplanted hearts is localised to the medial and adventitial layers of the coronary arteries and not in the intima. ${ }^{10}$ The relevance of links between CMV and post-transplant or postangioplasty lesions to the aetiology of primary coronary artery disease is therefore uncertain.

There is limited epidemiological evidence relating CMV specifically to primary coronary atheroma in immunocompetent individuals. ${ }^{8}$ Cytomegalovirus seropositivity was not independently related to coronary atherosclerosis in 314 hearts removed at transplantation, although a stronger and statistically significant association emerged in younger subjects. ${ }^{11}$ In a case-control study of angiographically proven coronary heart disease, $90 \%$ of cases and $86 \%$ of controls were seropositive for CMV, but fewer of the cases than controls had high CMV titres ( $14 \%$ v $21 \%$, respectively)..$^{12}$ Among control patients matched to surgically treated cases of atherosclerosis, high CMV antibody titres were more common in those who subsequently developed coronary events than in the remain$\operatorname{der}(45 \%$ v $26 \%)$, but this comparison was not adjusted for potential confounding variables. ${ }^{5}$ In a population based cohort of elderly men, ${ }^{13}$ CMV seropositivity at entry was of similar prevalence among those who subsequently developed coronary heart disease as among age matched controls ( $72 \% v 76 \%$, respectively).

In this paper we report findings from a nested case-control analysis relating CMV seropositivity to subsequent 10 year incidence of fatal and non-fatal ischaemic heart disease among middle aged men in south Wales.

\section{Methods}

In the Caerphilly prospective heart disease study, ${ }^{14} 2512$ men aged 45-59 years were 
Table 1 Cytomegalovirus (CMV) antibodies in incident cases of ischaemic heart disease and controls, and odds ratios compared with seronegative individuals

\begin{tabular}{lrcl}
\hline CMV serology & Cases & Controls & Odds ratio (95\% CI)* \\
\hline $\begin{array}{l}\text { All subjects } \\
\text { Seronegative }\end{array}$ & 31 & 36 & 1.00 (reference) \\
Seropositive & 164 & 180 & $1.06(0.63$ to 1.79$)$ \\
& & & \\
Seropositive by optical density & & \\
Lowest quarter & 43 & 43 & $1.16(0.61$ to 2.20$)$ \\
Second quarter & 34 & 51 & $0.77(0.41$ to 1.48$)$ \\
Third quarter & 40 & 47 & $0.99(0.52$ to 1.87$)$ \\
Highest quarter & 47 & 39 & $1.40(0.74$ to 2.66$)$ \\
\hline
\end{tabular}

${ }^{\star}$ Compared with seronegative individuals.

$\mathrm{CI}$, confidence interval.

recruited in the Caerphilly area of south Wales during 1979-83. Symptoms and ECG abnormalities suggestive of past or current ischaemic heart disease were ascertained and a range of cardiovascular risk factors measured, including smoking history, standing height, body weight, spirometry, blood pressure, total cholesterol, ${ }^{15}$ fibrinogen, plasma viscosity, and leucocyte count. ${ }^{16}$ Socioeconomic status was classified according to the registrar general's social class of current occupation and father's occupation during childhood. ${ }^{17}$

The sample has been followed up at five year intervals and the third round of fieldwork (phase III) was completed during 1989-93. Incident ischaemic heart disease was ascertained from death certificates and review of hospital notes and ECG changes, using the same conventions as in previous prospective analyses of this cohort. ${ }^{15} 16$

In all, 312 men died of ischaemic heart disease (ICD-9 codes 410-414) or developed non-fatal myocardial infarction during 10 years of follow up. These 312 cases were individually matched with controls of the same age at entry. Frozen plasma specimens banked at the entry (phase I) examination were available for 195 cases $(63 \%)$ and 216 controls $(69 \%)$. They had been stored at $-20^{\circ} \mathrm{C}$ since collection in 1979 83 , with one thaw cycle. The main reason for specimens being missing was depletion of material during previous seroepidemiological studies involving about one quarter of the cohort.

Table 2 Cardiovascular risk factors at entry among control subjects by cytomegalovirus seropositivity

\begin{tabular}{llll}
\hline Risk factor & $\begin{array}{l}\text { Seropositive } \\
(n=180)\end{array}$ & $\begin{array}{l}\text { Seronegative } \\
(n=36)\end{array}$ & $p$ Value $^{\star}$ \\
\hline Mean $(S D)$ & $53.6(4.3)$ & $52.4(4.3)$ & 0.14 \\
Age (years) & $1.71(0.07)$ & $1.71(0.07)$ & 0.88 \\
Standing height $(\mathrm{m})$ & $2.65(0.82)$ & $2.61(0.60)$ & 0.82 \\
FEV $($ litres) & $26.0(3.3)$ & $26.2(3.6)$ & 0.70 \\
Body mass index $\left(\mathrm{kg} / \mathrm{m}^{2}\right)$ & $141.0(20.1)$ & $143.4(18.3)$ & 0.50 \\
Systolic blood pressure $(\mathrm{mm} \mathrm{Hg})$ & $5.69(1.00)$ & $5.72(1.17)$ & 0.88 \\
Total cholesterol $(\mathrm{mmol} / \mathrm{l})$ & $3.77(0.76)$ & $3.54(0.59)$ & $0.13 \dagger$ \\
Fibrinogen $(\mathrm{g} / \mathrm{l})$ & $1.70(0.09)$ & $1.70(0.08)$ & $0.92 \dagger$ \\
Viscocity $(\mathrm{cP})$ & $6.97(2.14)$ & $6.55(1.74)$ & $0.27 \dagger$ \\
Leucocytes $\left(\times 10^{9} / \mathrm{l}\right)$ & & & \\
Number $(\%)$ & $151(84)$ & $28(78)$ & $0.47 \ddagger$ \\
Ever smoked & $72(40)$ & $15(42)$ & $0.98 \ddagger$ \\
Current cigarette smoker & $48(28)$ & $12(34)$ & $0.32 \coprod$ \\
Non-manual occupation & $12(7)$ & $5(15)$ & $0.16 \rrbracket$ \\
Father's occupation non-manual & & & \\
\hline
\end{tabular}

$\mathrm{FEV}_{1}$, forced expiratory volume in one second.

*Two sample $t$ test on untransformed data, unless stated otherwise.

tComparison by $t$ test on log transformed data.

$\ddagger$ Yates corrected $\chi^{2}$ test.

§Test for trend across six social classes (I, II, IIINM, IIIM, IV, V).
Presence of IgG antibodies to CMV was assessed by an in-house enzyme linked immunosorbent assay (ELISA), as described in detail elsewhere, ${ }^{18}{ }^{19}$ with confirmatory latex testing of borderline ELISA results.

Owing to the missing specimens, individual matching was preserved for only 130 sets of cases and controls. However, comparison of unmatched and matched pair analyses on these 130 case-control sets showed that the matched and unmatched odds ratios relating CMV seropositivity to incident ischaemic heart disease were very similar (to within $2 \%$ of each other). For this reason the individual matching was ignored, and results were analysed for all 195 cases and 216 controls, adjusting for age and other covariates by multiple logistic regression using STATA. ${ }^{20}$

These models were restricted to 395 subjects (96\% of 411 ) with complete data for age, smoking history, body mass index, systolic blood pressure, total cholesterol, and fibrinogen. Subjects with missing or inapplicable social class data were held in the model, using a separate category to designate missing social class currently $(\mathrm{n}=17)$ or in childhood $(n=49)$. Cytomegalovirus serology was analysed as a dichotomy (seropositive $v$ seronegative) and as a ordinal variable, grouping the seropositive men into four groups of equal size according to optical density on ELISA testing.

\section{Results}

The 195 cases comprised 94 men who died of ischaemic heart disease and 101 who developed myocardial infarction but did not die of ischaemic heart disease during the 10 years of follow up. Table 1 shows the relation between CMV IgG titre (as indicated by optical density) and incident ischaemic heart disease. Among the cases, $84 \%$ (164 of 195) were seropositive for CMV, compared with $83 \%$ (180 of 216) among the controls. There was no overall trend for incident cases to have higher optical densities than controls $\left(\chi^{2}\right.$ test for linear trend across optical density groups $=0.57, \mathrm{p}=0.45$ ), although men with the highest optical densities were at highest risk of ischaemic heart disease.

The crude odds ratio relating CMV seropositivity to incident ischaemic heart disease was 1.06 (95\% confidence interval (CI) 0.63 to 1.79). Separate analysis of the 130 matched pairs yielded a corresponding odds ratio of 1.74 (95\% CI 0.90 to 3.38 ) by unmatched analysis and 1.71 ( 0.85 to 3.59$)$ by matched analysis. However, among the 65 unmatched cases and 86 unmatched controls, the association of CMV and ischaemic heart disease was in the opposite direction (odds ratio 0.43, 95\% CI 0.17 to 1.06 ). Although these odds ratios differ significantly, no obvious explanation could be found for the effect modification between matched and unmatched subsets. For this reason, we assumed that the difference arose by chance and all data were aggregated in further analyses.

Table 2 shows the relation between the principal cardiovascular risk factors and CMV antibody status among the controls. None of the associations was significant at the $10 \%$ level. 
Among 395 subjects (186 cases and 209 controls) with complete covariate data, the unadjusted odds ratio relating CMV seropositivity to incident ischaemic heart disease was 1.13 (95\% CI 0.66 to 1.92 ). After adjustment for age, smoking history, body mass index, systolic blood pressure, total cholesterol, and social class currently and in childhood, the odds ratio was almost identical $(1.11,95 \%$ CI 0.63 to 1.97 ). Further adjustment for fibrinogen reduced the odds ratio to $0.96(95 \%$ CI 0.53 to 1.71 ).

\section{Discussion}

Serological evidence of cytomegalovirus infection is highly prevalent in general population samples. In our study, five of six men were seropositive for CMV at entry. This is higher than the figure of $60 \%$ considered typical of developed countries ${ }^{21}$ (based on data from blood donors ${ }^{22}$ ), but similar to the seroprevalence reported among women of the same age range in south Wales. ${ }^{23}$

Ossewaarde et al used a nested case-control design similar to ours to compare 54 incident cases of ischaemic heart disease with 108 age matched controls drawn from a cohort of men aged 65 and over in Zutphen, the Netherlands. ${ }^{13}$ CMV IgG antibodies were detected in $72 \%$ of cases and $76 \%$ of controls, a slightly lower seroprevalence than we found in Caerphilly. The odds ratio for incident ischaemic heart disease in the Zutphen study was 0.8 (95\% CI 0.4 to 1.7), similar to our result.

Cour et al compared 73 patients with angiographically proven coronary heart disease and 73 controls matched for age, sex, and social background, and found that $90 \%$ of cases and $86 \%$ of controls were seropositive for CMV. ${ }^{12}$ This corresponds to an unmatched odds ratio of 1.5 (95\% CI 0.5 to 4.2 ). High CMV titres were less prevalent among the cases $(14 \%)$ than the controls $(21 \%)$, yielding an unmatched odds ratio of 0.6 (95\% CI 0.3 to 1.5). In contrast, Adam et al reported a significant positive association of high CMV titres and incident ischaemic heart disease over five years among control patients recruited to a case-control study of peripheral atherosclerosis and CMV infection ( $45 \% v 26 \%$, odds ratio $2.4,95 \%$ CI 1.2 to 4.9$).^{5}$

In our nested case-control analysis the incidence of myocardial infarction over 10 years of follow up was similar in men with and without CMV infection at entry (odds ratio 1.1, 95\% CI 0.6 to 1.8 ). The odds ratio comparing men with the highest optical densities with all other men was 1.4 (95\% CI 0.9 to 2.3). The confidence intervals for these results overlap with those of previous studies ${ }^{512}{ }^{13}$ and all four studies would be consistent with a weak association of CMV infection and primary coronary atheroma. However, a twofold or greater increase in ischaemic heart disease risk among seropositive men appears unlikely.

Previous studies have not reported the association of CMV seropositivity with cardiovascular risk factors. No strong associations emerged among our control subjects, suggesting that major coronary risk factors - such as age, smoking, blood pressure, and circulating lipid levels-are unlikely to confound the association of CMV and ischaemic heart disease. This is confirmed by the similarity of odds ratios before and after adjustment for these potential confounding variables.

In this study we have shown the potential for rapid epidemiological evaluation of the association of CMV infection with ischaemic heart disease risk using stored samples from prospective cohort studies. Although a strong relation is improbable, the prevalence of infection is high, implying that even a weak association may be of public health importance. Further epidemiological studies of clinical complications from primary coronary atheroma are required in order to complement the emerging evidence from pathological and postoperative studies, and to evaluate more precisely the risks related to persistent cytomegalovirus infection in the general population.

We are deeply indebted to the men of Caerphilly who participated in the multiple follow up examinations, and to many unnamed field workers who collected data and specimens during phases I to III of the study. We are also particularly during phases I to III of the study. We are also particularly attention to specimen handling and laboratory analyses. The study was funded by the British Heart Foundation.

1 Melnick JL, Petrie BL, Dreesman GR, et al. Cytomegalovirus antigen within human arterial smooth muscle cells. virus antigen within het 1983;ii:644-7.

2 Hendrix MGR, Salimans MMM, van Boven CPA, et al. High prevalence of latently present cytomegalovirus in arterial walls of patients suffering from grade III atherosclerosis. Am f Pathol 1989;136:23-8.

3 Melnick JL, Hu C, Burek J, et al. Cytomegalovirus DNA in arterial walls of patients with atherosclerosis. $f \mathrm{Med}$ Virol 1994;42:170-4

4 Hendrix MG, Daemen M, Bruggeman CA. Cytomegalovius nucleic acid distribution within the human vascular tree. Am f Pathol 1991;138:563-7.

5 Adam E, Melnick JL, Probtsfield JL, et al. High levels of cytomegalovirus antibody in patients requiring vascular

6 Sorgery for atherosclerosis. Lancet 1987;ii:291-3. herpesvirus and carotid atherosclerosis: the ARIC study. $\mathscr{f}$ Med Virol 1994;42:33-7.

7 Nieto FJ, Adam E, Sorlie P, et al. Cohort study of cytomegalovirus infection as a risk factor for carotid intimal thickening, a measure of subclinical atherosclerosis. Circulation 1996;94:922-7.

8 Danesh J, Collins R, Peto R. Chronic infections and coronary heart disease: is there a link? Lancet 1997;350:430-6.

9 Speir E, Modali R, Huang ES, et al. Potential role of human cytomegalovirus and p53 interaction in coronary restenosis. Science 1994;265:391-4.

10 Gulizia JM, Kandolf R, Kendall TJ, et al. Infrequency of cytomegalovirus genome in coronary arteriopathy of human heart allografts. Am F Pathol 1995;147:461-75.

11 Dummer S, Lee A, Breinig MK, et al. Investigation of cytomegalovirus infection as a risk factor for coronary atheosclerosis in the explanted hearts of patients undergoing heart transplantation. F Med Virol 1994;44:305-9.

12 Cour MI, Lopez de Atalaya FJ, Palau L, et al. Lack of serological association between herpesvirus and atherosclerosis. Lancet 1989;i:279.

13 Ossewaarde JM, Feskens EJM, de Vries A, et al. Chlamydia pneumoniae is a risk factor for coronary heart disease in symptom-free elderly men, but Helicobacter pylori and symptom-free elderly men, but Helicobacter pylori and 14 The Caerphilly and Speedwell Collaborative Group. The Caerphilly and Speedwell collaborative heart disease studies. $\mathcal{F}$ Epidemiol Community Health 1984;38:259-62.

15 Bainton D, Miller NC, Bolton CH, et al. Plasma triglyceride and high density lipoprotein cholesterol as predictors of schaemic heart disease in British men. Br Heart f 1992;68: 60-6.

16 Yarnell JWG, Baker IA, Sweetnam PM, et al. Fibrinogen, viscosity and white blood cell count are major risk factors for ischaemic heart disease. The Caerphilly and Speedwell collaborative heart disease studies. Circulation 1991;83: 836-44.

17 Office of Population Censuses and Surveys. Classification of occupations. London: HMSO, 1980.

18 Booth JC, Hannington G, Aziz TAG, et al. Comparison of enzyme-linked immunosorbent assay (ELISA) technique and complement-fixation test for estimation of cytomegalovirus IgG antibody. F Clin Pathol 1979;32:122-7.

19 Booth JC, Hannington G, Bakir TMF, et al. Comparison of enzyme-linked immunosorbent assay, radioimmunoassay, complement fixation, anticomplement immunofluores- 
cence and passive haemagglutination techniques for cence and passive haemagglutination techniques for detecting cytom

20 Stata Corporation. STATA reference manual: release 3.1, 6th ed. College Station, Texas: Stata Corporation, 1993.

21 Griffiths PD. Chronic cytomegalovirus infection. $\mathcal{f}$ Virol Methods 1988;21:79-86.
22 Krech U. Complement-fixing antibodies against cytomegaKrech U. Complement-fixing antibodies against cytomegalovirus in

23 Yarnell JWG, Milbank JE, Munro JA, et al. The prevalence of cytomegalovirus antibody in women: an epidemioogical study from south Wales. Public Health 1982;96: $251-5$.

\section{IMAGES IN CARDIOLOGY}

\section{A tilting valve}

A 38 year old man was admitted to hospital because of fatigue and progressive dyspnoea. $\mathrm{He}$ had a prosthetic aortic valve implanted at age 22 years because of aortic regurgitation owing to bacterial endocarditis. Physical examination revealed a systolic and diastolic murmur. Chest radiography showed an abnormal position of the prosthetic aortic valve. Cineradiography was done because of suspicion of aortic valve detachment. Two consecutive frames are shown

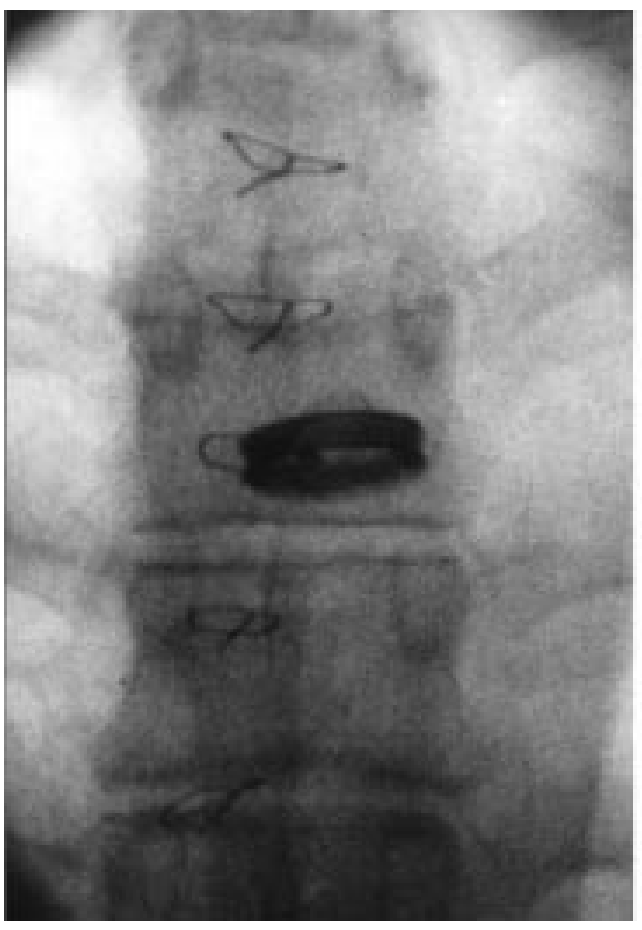

from of an antero-posterior view. The left panel shows the valve in the aortic position, the next frame (right panel) shows the entire valve is displaced during early systole.

The patient was operated shortly after the cineradiography. The aortic valve ring was hanging loose and connected with just two remaining sutures. He received a new valve and was doing well six months after the operation.

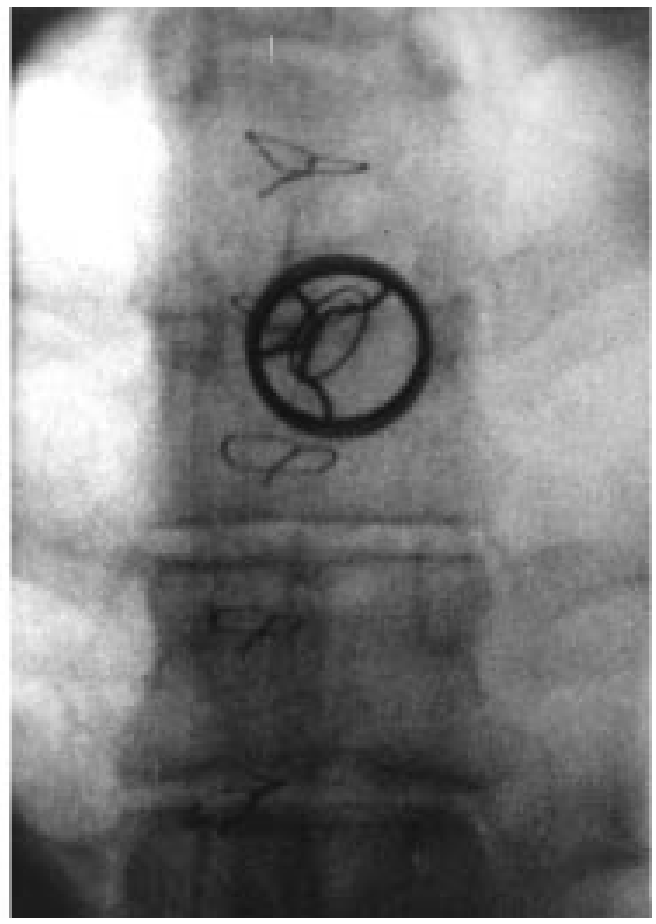

R A TIO

H J G M CRIJNS 